\title{
Interaction of miR-181b and IFNA1 Polymorphisms on the Risk of Systemic Lupus Erythematosus
}

\author{
Yong-Ling He $\mathbb{D}^{\mathrm{D}}$, Jun Yang, Zhi-Neng Zeng, and Xiang Shi \\ Department of Laboratory Medicine, Affiliated Hospital of Guilin Medical University, Guilin, Guangxi, China \\ Correspondence should be addressed to Yong-Ling He; glhyl126@126.com
}

Received 19 January 2020; Accepted 16 April 2020; Published 25 April 2020

Academic Editor: Takahisa Gono

Copyright (C) 2020 Yong-Ling He et al. This is an open access article distributed under the Creative Commons Attribution License, which permits unrestricted use, distribution, and reproduction in any medium, provided the original work is properly cited.

\begin{abstract}
Introduction. A previous work has discovered that chromosome 1q32 locus linked to the risk of systemic lupus erythematosus (SLE) and miR-181b located on the susceptibility site with downregulation inversely correlating to its target molecular interferon alpha 1 (IFNA1). The purpose of this study was to investigate the association of miR-181b and IFNA1 polymorphisms with IS risk. Methods. The miR-181b rs322931, IFNA1 rs1332190, and rs10811543 were genotyped using a Multiplex SNaPshot assay. miR$181 \mathrm{~b}$ expression levels in plasma of SLE patients and controls were analyzed using quantitative PCR. Results. The rs 322931 CT, $\mathrm{CT} / \mathrm{TT}$, and $\mathrm{T}$ allele exerted an increased trend of SLE risk (CT vs. CC: adjusted OR $=1.71,95 \%$ CI 1.16-2.50, $P=0.01$; CT/TT vs. $\mathrm{CC}$ : adjusted $\mathrm{OR}=1.45,95 \% \mathrm{CI} 1.08-1.95, P=0.01$; T vs. C: adjusted $\mathrm{OR}=1.38,95 \%$ CI $1.07-1.79, P=0.01)$. Combined genotypes of the rs322931 CT/TT+rs1332190 TT and the rs322931 CC+rs10811543 AG/AA also revealed an increased risk of SLE. Gene-gene interaction analysis showed that a three-locus model consisting of rs322931, rs1332190, and rs10811543 attributed an increased risk of SLE. Further genotype-phenotype analysis revealed that rs322931 CT/TT carriers displayed lower levels of miR-181b. Conclusions. These findings indicate that the miR-181b rs322931 may be singly and jointly responsible for the etiology of SLE by altering miR-181b expression.
\end{abstract}

\section{Introduction}

Systemic lupus erythematosus (SLE), a chronic inflammatory disease characterized by multiple immunologic abnormalities, can damage many organs [1]. The prevalence rates of SLE are about 17-48/100,000 population worldwide, and females are 3-6 times more frequently affected than males. The burden of SLE is not only physical and mental health but also socioeconomic impact because the most common age of onset is 20-40 years, and patients with that age are still raising or supporting families [2]. Risk factors of SLE included cigarette smoking, oxidative stress, ultraviolet light, infection, and hormonal action as well as genetic factors [3-12]. Environmental exposure may trigger SLE in individuals who carry a predisposing background of genetic susceptibility $[2,4,7]$. Several single-nucleotide polymorphisms (SNPs) in coding genes have been found to be involved in the pathogenesis of SLE, such as rs1051169 in S100B [8]; rs20541 in interleukin- (IL-) 13 [9]; rs11556218, rs4778889, and rs4072111 in $I L-16$ [10]; rs2227513 in IL-22 [11]; and rs7977932 in $I L-31$ [12].

In addition to coding genes, noncoding transcripts, such as microRNAs (miRNAs) also play a critical role in modulating immune response of SLE [13-16]. Until today, more than 1500 miRNAs have been sequenced in human [17], with an estimation of regulating over one-third of genome expression [18]. Among them, the miR-181 family was found to be downregulated in patients with SLE, being an important modulator of $\mathrm{B}$ and $\mathrm{T}$ cell differentiation and inflammatory reaction, which were key events in the initiation and development of SLE [13, 19-22].

Recently, miRNA-related genetic variant has been reported to be a risk factor for a series of human diseases, including SLE [23-30]. For example, an SNP rs4937333 T allele was reported to be associated with a significantly increased risk of SLE by enhancing the binding of miR5003 to transcriptional factor ETS1 and decreasing ETS1 expression [26]. A genetic variant of rs322931 was reported 
to alter both transcriptional activity and the expression of miR-181b $[29,30]$. To date, no study reported the association of miR-181b rs322931 with the risk of SLE. In this hospitalbased case-control study, we investigated whether the miR$181 \mathrm{~b}$ rs322931 was related to the occurrence of SLE in a Chinese Han population. As miR-181b may contribute to the development and progression of SLE through directly targeting molecular interferon alpha 1 (IFNA1) [13], two SNPs (i.e., rs1332190 and rs10811543) in the promoter region of IFNA1 were also examined for gene-gene interaction analysis. We found that the miR-181b rs322931 and IFNA1 rs1332190 and rs10811543 may play an interactive role in the etiology of SLE.

\section{Materials and Methods}

2.1. Study Population. A total of 402 SLE patients and 430 age- and sex-matched healthy controls were included in this study. Patients with SLE were consecutively enrolled from the Affiliated Hospital of Guilin Medical University between May 2012 and December 2018. All patients fulfilled the American College of Rheumatology criteria for SLE (1997) [31]. The controls were also registered from the same hospital at the same period. We excluded those controls if they had autoimmune diseases, such as malar butterfly rash, photosensitivity, fever, erythra, and edema. All the subjects without blood transfusion were genetically unrelated ethnic Han Chinese living in Guangxi province. After written informed consent was signed, each participant donated $3-5 \mathrm{~mL}$ of eathylene diamine tetraacetic acid-anticoagulated peripheral venous blood. Plasma was separated by centrifuging for $10 \mathrm{~min}$ at $1000 \mathrm{rpm}$ and stored in $-80^{\circ} \mathrm{C}$ until analysis. The study protocol was approved by the Institutional Review Board of the Affiliated Hospital of Guilin Medical University.

2.2. SNP Selection. We selected miR-181b rs322931 locating on the chromosome 1q32 that has been identified to be a risk locus of SLE [32]. Additionally, we selected SNPs within the IFNA1 that is a target gene of miR-181b [32], with the inclusion criteria of minor allele frequency $>10 \%$ in Chinese Han population. Two SNPs (i.e., rs1332190 and rs10811543) in the promoter of IFNA1 were selected.

2.3. Genotyping. Genomic DNA was extracted from leukocytes of peripheral blood using the commercially available kit (Tiangen, Beijing, China). The miR-181b rs322931 and IFNA1 rs1332190 and rs10811543 were genotyped using a Multiplex SNaPshot assay. For quality control, negative control replacing template DNA with distilled water was used in each run and about $5 \%$ of all samples were randomly selected for validation using DNA sequencing. The results between the two genotyping methods were concordant.

2.4. Quantitative PCR ( $q P C R$ ) of $m i R-181 b$. Total RNA was isolated from plasma using a commercial kit (Qiagen, Hilden, Germany) following the manufacturer's protocol. cDNA was generated using the Mir-X miRNA First-Strand Synthesis Kit (Takara Bio USA, Mountain View, CA, USA) according to the manufacturer's manual. miR-181b levels in cases and controls were quantified using the Mir-X miRNA qRT-PCR
TB Green Kit (Takara Bio USA). qPCR was run on an ABI 7900HT real-time PCR machine (Applied Biosystems, CA, USA). U6 was used as an internal control, and relative expression of miR-181b was determined using the $2^{-\Delta \Delta \mathrm{Ct}}$ method [33].

2.5. Statistical Analysis. Statistical analysis was done using SPSS software version 13.0 (SPSS, Chicago, IL, USA). The $\chi^{2}$ test was used to evaluate Hardy-Weinberg equilibrium (HWE) and the differences of the rs322931, rs1332190, and rs10811543 between cases and controls. Odds ratios (ORs) and $95 \%$ confidence intervals (CIs) were used to estimate the association between each SNP and SLE risk using a logistic regression model after adjustment for age and gender. Linkage disequilibrium (LD) and haplotype analysis were performed using the SHEsis software [34]. The statistical significant value of SNPs was set as $5 \times 10^{-8}$ that was used in genome-wide association study. Gene-gene interaction analysis was performed by using multifactor dimensionality reduction (MDR) platform [35]. miR-181b expression levels were compared using the Mann-Whitney $U$ test, and a value of $P<0.05$ was considered statistically significant.

\section{Results}

3.1. Characteristics of Study Population. The characteristics of the study population are summarized in Table 1 . There were no significant differences in the distribution of age and gender $(P=0.27$ and 0.10 , respectively). Approximately half of the SLE patients had photosensitivity, leucopenia, anaemia, complement depression, renal disorder, and arthritis, while only about $30 \%$ patients had malar rash and negative AdsA. Most of the patients (87.3\%) had positive ANA.

3.2. Main Effects of SNPs in miR-181b and IFNA1 on SLE Risk. The genotype and allelic frequencies of the selected SNPs among cases and controls are presented in Table 2. The genotype distributions of the miR-181b rs322931 and IFNA1 rs1332190 and rs10811543 met the HWE requirements in both cases and controls $(P>0.05)$. Compared to the miR-181b rs322931 CC genotype, individuals with the CT variant genotype exerted an increased trend of SLE risk (adjusted OR $=1.71,95 \%$ CI 1.16-2.50, $P=0.01$ ). Under the dominant genetic of inheritance, carriers with the CT/TT genotypes had a 1.45-fold increased risk of SLE (adjusted OR $=1.45,95 \%$ CI 1.08-1.95, $P=0.01$ ). Moreover, carriers with the $\mathrm{T}$ allele had a 1.38-fold increased risk of SLE (adjusted OR $=1.38,95 \%$ CI 1.07-1.79, $P=0.01$ ). For the rs1332190 and rs10811543, no significant difference between SLE patients and controls was found $(P>0.05)$. Stratification analysis also showed null association between the three SNPs and clinical features of SLE (Table 3).

3.3. Haplotype and Combined Analysis. LD results revealed that the rs1332190 and rs10811543 were in strong LD $\left(\mathrm{D}^{\prime}=0.97, r^{2}=0.67\right)$. The frequencies of 4 possible haplotypes are shown in Table 4 . There was no significant difference of the haplotype between cases and controls. 
TABLE 1: Characteristics of the study population.

\begin{tabular}{|c|c|c|c|}
\hline Variables & Controls, $n=430$ & Patients with SLE, $n=402$ & $P$ value \\
\hline Age $($ mean $\pm S D)$ & $34.3( \pm 12.2)$ & $33.2( \pm 16.0)$ & 0.27 \\
\hline Male/female (\%) & $105(24.4) / 325(75.6)$ & $79(19.7) / 323(80.3)$ & 0.10 \\
\hline Malar rash $(\%)$ & & $123(30.6)$ & \\
\hline Photosensitivity (\%) & & $241(60.0)$ & \\
\hline Leucopenia (\%) & & $214(53.2)$ & \\
\hline Anaemia (\%) & & $224(55.7)$ & \\
\hline Complement depression (\%) & & $264(65.8)$ & \\
\hline Renal disorder (\%) & & $205(51.0)$ & \\
\hline Arthritis (\%) & & $235(58.5)$ & \\
\hline ANA (\%) & & $351(87.3)$ & \\
\hline AdsA (\%) & & $272(67.7)$ & \\
\hline
\end{tabular}

SLE: systemic lupus erythematosus; SD: standard deviation; ANA: antinuclear antibodies; AdsA: anti-double stranded DNA antibody.

TABLE 2: Association between SNPs in miR-181b and IFNA1 and risk of SLE.

\begin{tabular}{lccc}
\hline Polymorphisms & Controls, $n=430(\%)$ & SLE, $n=402(\%)$ & ${\text { Adjusted OR }(95 \% \text { CI })^{\dagger}}^{P \text { value }}$ \\
\hline miR-181b rs322931 & $310(72.1)$ & $257(63.9)$ & 1.00 \\
CC & $114(26.5)$ & $135(33.6)$ & $1.71(1.16-2.50)$ \\
CT & $120(27.9)$ & $145(36.1)$ & $1.45(1.08-1.95)$ \\
CT/TT & $734(85.3)$ & $649(80.7)$ & 1.00 \\
C allele & $126(14.7)$ & $155(19.3)$ & $1.38(1.07-1.79)$ \\
T allele & & & 1.00 \\
IFNA1 rs1332190 & $220(51.2)$ & $199(49.5)$ & $1.02(0.77-1.36)$ \\
TT & $185(43.0)$ & $168(41.8)$ & $1.08(0.83-1.42)$ \\
CT & $210(48.8)$ & $563(50.5)$ & 1.00 \\
CT/CC & $625(72.7)$ & $238(29.6)$ & $1.13(0.91-1.40)$ \\
T allele & $235(27.3)$ & & 0.01 \\
C allele & & $250(62.2)$ & 0.89 \\
IFNA1 rs10811543 & $260(60.5)$ & $132(32.8)$ & 0.56 \\
GG & $146(34.0)$ & $152(37.8)$ & $0.96(0.71-1.28)$ \\
AG & $170(39.5)$ & $632(78.6)$ & $0.95(0.71-1.25)$ \\
AG/AA & $666(77.4)$ & $172(21.4)$ & 1.00 \\
G allele & $194(22.6)$ & $0.95(0.75-1.20)$ \\
A allele & & & 0.76 \\
\hline
\end{tabular}

SNP: single-nucleotide polymorphism; IFNA1: interferon alpha 1; SLE: systemic lupus erythematosus; OR: odds ratio; CI: confidence interval. ${ }^{\dagger}$ Adjusted by age and gender.

Compared to the combined genotypes rs322931 CC + rs1332190 TT, the combined genotypes rs322931 CT/TT +rs1332190 TT and rs322931 CC+rs1332190 CT/CC were associated with a reduced risk of SLE $(\mathrm{OR}=0.06,95 \% \mathrm{CI}$ $0.02-0.18, \quad P=2.55 \times 10^{-11} ;$ adjusted $\mathrm{OR}=0.37, \quad 95 \% \mathrm{CI}$ $0.26-0.53, P=3.74 \times 10^{-8}$, respectively). Compared to the combined genotypes rs322931 CC+rs10811543 GG, the combined genotypes rs322931 CC+rs10811543 AG/AA were associated with a reduced risk of SLE $(\mathrm{OR}=0.33,95 \% \mathrm{CI}$ 0.22-0.49, $P=3.07 \times 10^{-8}$ ) (Table 5).

3.4. Interaction Analysis. Table 6 shows interaction analysis among the miR-181b rs322931 and IFNA1 rs1332190 and rs10811543. When taking the three SNPs together, the accu- racy was the highest with the cross-validation consistency of $10 / 10(\mathrm{OR}=9.99,95 \%$ CI 6.54-15.26, $P<0.001)$, indicating that the miR-181b rs322931 and IFNA1 rs1332190 and rs10811543 were interactively associated with the risk of SLE.

3.5. The rs322931 CT/TT Exhibited Lower Levels of miR-181b. The expression levels of miR-181b were analyzed using qPCR in controls $(n=44)$ and SLE patients $(n=42)$. As shown in Figure 1(a), the miR-181b expression was lower in SLE patients compared to controls $(P<0.05)$. When comparing the relationship of the rs322931 to miR-181b expression, we found that the rs322931 CT/TT carriers had lower levels of miR-181b than rs322931 CC carriers $(P<0.05)$ (Figures 1(b) and 1(c)). 
TABLE 3: Stratification analyses of SNPs in miR-181b and IFNA1 and clinical features of SLE.

\begin{tabular}{|c|c|c|c|c|c|c|}
\hline \multirow[t]{2}{*}{ Variables } & \multicolumn{2}{|c|}{$\begin{array}{c}\text { miR-181b rs322931 } \\
\text { CT/TT vs. CC }\end{array}$} & \multicolumn{2}{|c|}{$\begin{array}{c}\text { IFNA1 rs } 1332190 \\
\text { CT/CC vs. TT }\end{array}$} & \multicolumn{2}{|c|}{$\begin{array}{c}\text { IFNA1 rs } 10811543 \\
\text { AG/AA vs. GG }\end{array}$} \\
\hline & Adjusted $\mathrm{OR}^{\dagger}$ & $P$ value & Adjusted $\mathrm{OR}^{\dagger}$ & $P$ value & Adjusted $\mathrm{OR}^{\dagger}$ & $P$ value \\
\hline Malar rash & $1.18(0.75-1.85)$ & 0.47 & $1.09(0.71-1.67)$ & 0.70 & $1.29(0.82-2.02)$ & 0.26 \\
\hline Photosensitivity & $1.18(0.75-1.85)$ & 0.20 & $0.61(0.40-0.91)$ & 0.02 & $0.78(0.52-1.19)$ & 0.25 \\
\hline Leucopenia & $0.80(0.53-1.21)$ & 0.30 & $0.75(0.51-1.12)$ & 0.16 & $0.88(0.59-1.32)$ & 0.54 \\
\hline Anaemia & $0.99(0.66-1.49)$ & 0.96 & $1.17(0.79-1.74)$ & 0.43 & $1.07(0.71-1.60)$ & 0.76 \\
\hline Complement depression & $1.10(0.71-1.68)$ & 0.67 & $1.05(0.69-1.58)$ & 0.83 & $1.03(0.67-1.58)$ & 0.89 \\
\hline Renal disorder & $1.13(0.75-1.71)$ & 0.55 & $1.36(0.92-2.01)$ & 0.13 & $1.13(0.75-1.69)$ & 0.56 \\
\hline Arthritis & $0.75(0.50-1.15)$ & 0.19 & $0.92(0.62-1.37)$ & 0.68 & $1.06(0.70-1.59)$ & 0.80 \\
\hline ANA & $0.87(0.47-1.62)$ & 0.66 & $0.85(0.47-1.52)$ & 0.58 & $0.71(0.38-1.34)$ & 0.28 \\
\hline AdsA & $1.10(0.71-1.70)$ & 0.67 & $1.34(0.88-2.04)$ & 0.18 & $1.10(0.72-1.70)$ & 0.66 \\
\hline
\end{tabular}

SNP: single-nucleotide polymorphism; IFNA1: interferon alpha 1; SLE: systemic lupus erythematosus; OR: odds ratio; CI: confidence interval. ${ }^{\dagger}$ Adjusted by age and gender.

TABLE 4: Haplotype analysis of SNPs in IFNA1 between cases and controls.

\begin{tabular}{|c|c|c|c|c|c|}
\hline rs1332190 & rs10811543 & Controls, $n(\%)$ & SLE, $n(\%)$ & OR $(95 \% \mathrm{CI})$ & $P$ value \\
\hline $\mathrm{T}$ & G & $619(72.0)$ & $566(70.4)$ & 1.00 & \\
\hline $\mathrm{C}$ & A & $188(21.9)$ & $172(21.4)$ & $1.00(0.79-1.27)$ & 1.00 \\
\hline $\mathrm{C}$ & G & $47(5.5)$ & $66(8.2)$ & $1.54(1.04-2.27)$ & 0.03 \\
\hline $\mathrm{T}$ & A & $6(0.7)$ & $0(0.0)$ & - & - \\
\hline
\end{tabular}

SNP: single-nucleotide polymorphism; IFNA1: interferon alpha 1; SLE: systemic lupus erythematosus; OR: odds ratio; CI: confidence interval.

TABLE 5: Combined analyses of SNPs in miR-181b and IFNA1 and risk of SLE.

\begin{tabular}{|c|c|c|c|c|}
\hline Combined genotypes & Controls, $n=430(\%)$ & SLE, $n=402(\%)$ & OR $(95 \% \mathrm{CI})$ & $P$ value \\
\hline \multicolumn{5}{|l|}{ miR-181b rs322931-IFNA1 rs1332190 } \\
\hline rs322931 CC+rs1332190 TT & $166(38.6)$ & $195(48.5)$ & 1.00 & \\
\hline rs322931 CT/TT+rs1332190 TT & $54(12.6)$ & $4(1.0)$ & $0.06(0.02-0.18)$ & $2.55 \times 10^{-11}$ \\
\hline rs322931 CC+rs1332190 CT/CC & $144(33.5)$ & $62(15.4)$ & $0.37(0.26-0.53)$ & $3.74 \times 10^{-8}$ \\
\hline rs322931 CT/TT+rs1332190 CT/CC & $66(15.3)$ & $141(35.1)$ & $1.82(1.27-2.60)$ & 0.001 \\
\hline \multicolumn{5}{|l|}{ miR-181b rs322931-IFNA1 rs10811543 } \\
\hline rs322931 CC+rs10811543 GG & $196(45.6)$ & $216(53.7)$ & 1.00 & \\
\hline rs322931 CT/TT+rs10811543 GG & $64(14.9)$ & $34(8.5)$ & $0.48(0.31-0.76)$ & 0.002 \\
\hline rs322931 CC+rs10811543 AG/AA & $114(26.5)$ & $41(10.2)$ & $0.33(0.22-0.49)$ & $3.07 \times 10^{-8}$ \\
\hline rs322931 CT/TT+rs10811543 AG/AA & $56(13.0)$ & $111(27.6)$ & $1.80(1.24-2.62)$ & 0.002 \\
\hline
\end{tabular}

SNP: single-nucleotide polymorphism; IFNA1: interferon alpha 1; SLE: systemic lupus erythematosus; OR: odds ratio; CI: confidence interval.

TABLE 6: Interaction analysis of SNPs in miR-181b and IFNA1 and risk of SLE.

\begin{tabular}{|c|c|c|c|c|}
\hline Best candidate models & Accuracy & Cross-validation consistency & OR $(95 \% \mathrm{CI})$ & $P$ value \\
\hline rs322931 & 0.55 & $10 / 10$ & $1.46(1.09-1.95)$ & 0.01 \\
\hline rs322931/rs1332190 & 0.67 & $10 / 10$ & $9.53(6.24-14.55)$ & $<0.001$ \\
\hline rs322931/rs1332190/rs10811543 & 0.67 & $10 / 10$ & $9.99(6.54-15.26)$ & $<0.001$ \\
\hline
\end{tabular}

SNP: single-nucleotide polymorphism; IFNA1: interferon alpha 1; SLE: systemic lupus erythematosus; OR: odds ratio; CI confidence interval.

\section{Discussion}

In this hospital-based case-control study, we found that the miR-181b rs322931 C but not IFNA1 rs1332190 and rs10811543 was associated with an increased trend of SLE risk, whereas combined analysis showed a significant association of rs322931-rs1332190 and rs322931-rs10811543 with SLE risk. Gene-gene interaction analysis also showed that 


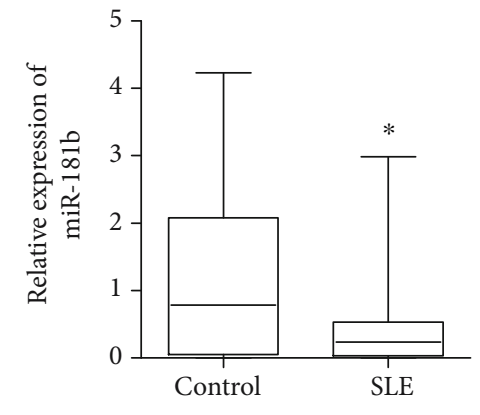

(a)

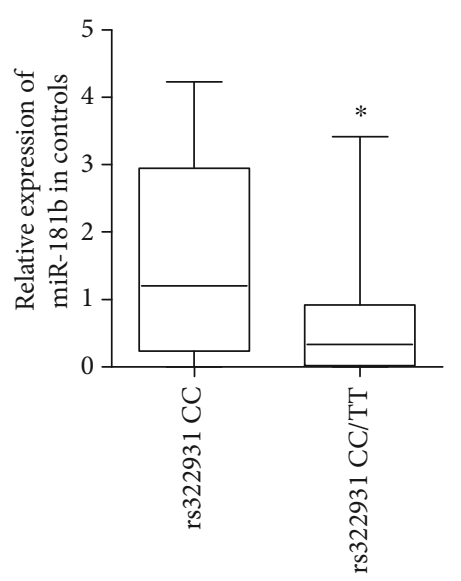

(b)

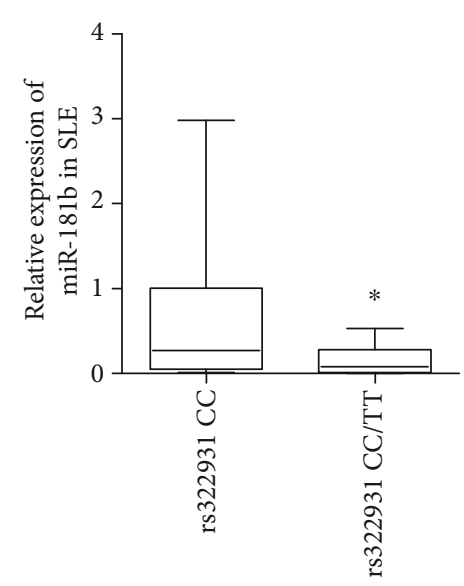

(c)

Figure 1: The rs322931 CT/TT exhibited lower levels of miR-181b. (a) Relative expression levels of miR-181b in controls ( $n=44)$ and SLE patients $(n=42)$. (b, c) The association of the rs322931 with miR-181b expression in controls and SLE patients $\left({ }^{*} P<0.05\right)$.

the three-locus model consisting of rs322931, rs1332190, and rs10811543 attributed an increased risk of SLE. In addition, genotype-phenotype analysis revealed that the rs322931 CT/TT carriers displayed lower levels of miR-181b. Our data shed light on the importance of miR-181b rs322931 in the setting of SLE.

The miR-181 family including miR-181a, miR-181b, miR-181c, and miR-181d is a critical regulator in the pathogenesis of SLE [13-16, 19, 36, 37]. Altered expression of miR181a was not only observed in SLE and lupus nephritis patients but also correlated to clinical features, such as the erythrocyte sedimentation rate, $\mathrm{C}$ reactive protein, antidsDNA antibody, complements, and score of SLE disease activity index [14-16, 36, 37]. miR-181b was also reported to be differentially expressed in SLE patients [13]. These findings indicate that miR-181 may be a biomarker for the diagnosis of SLE and monitor of SLE activity.

Previously, an SNP rs322931 was found to be a risk factor for positive emotion, reward processing, and ischaemic stroke through influencing transcriptional activity and the expression levels of miR-181b [29, 30, 38]. The current work has discovered that chromosome 1q32 locus linked to the risk of SLE and miR-181b located on the susceptibility site with the downregulation inversely correlating to its target molecular IFNA1 [32]. Based on this background, we hypothesized that the rs322931 may affect individual's susceptibility to SLE. Our findings in this association study confirmed this hypothesis. We found that the rs322931 CT/TT genotypes and T allele carriers had a 1.45 -fold and 1.38-fold increased risk of SLE, respectively. qPCR was then performed to detect the expression of miR-181b, and we found that the miR-181b levels were significantly lower in SLE patients than controls, confirming previous report [32]. Of note, after comparing the correlation of the rs322931 to miR-181b expression, we found that carriers with the rs322931 CT/TT genotypes exhibited lower levels of miR-181b in both SLE patients and controls. Taken together, we may conclude that the rs322931 CT/TT genotypes and T allele contributed to the risk of SLE by decreasing the levels of miR-181b.
In addition to the miR-181b rs322931, we examined much more SNPs in this study because SLE is a complex disease and involved in more than one gene. Genome-wide association study has provided evidence of susceptibility loci of SLE in the interferon (IFN) signaling pathway [39]. IFN- $\alpha$ is highly expressed and has emerged as a key pathogenic cytokine in SLE $[40,41]$. For example, IFN- $\alpha$ in sera from active SLE patients can induce differentiation of dendritic cells that capture and present antigens to CD4+ T cells [42]. IFN- $\alpha$ also exerts stimulatory effects on the adaptive immune system by enhancing $B$ cell differentiation and survival of autoimmune B cells [43, 44]. Moreover, IFN- $\alpha$ impairs autophagic degradation of mtDNA and vasculogenesis in SLE, serving as a drug target $[45,46]$. IFN- $\alpha$ is encoded by IFNA1 that is a target gene of miR-181b [13]. Genetic polymorphisms in IFNA1, therefore, were analyzed in this study. Although no significant association of the rs1332190 and rs10811543 in the promoter of IFNA1 with SLE risk was observed in single site analysis, combined analysis revealed an association of the 2 SNPs with SLE occurrence. Both the rs322931 CT/TT+rs1332190 CT/CC and the rs322931 CC+rs10811543 AG/AA were associated with a reduced risk of SLE. Finally, we performed miR-181b/IFNA1 interaction analysis, and we found that the miR-181b rs322931/rs1332190/rs10811543 was the best candidate model with the accuracy of 0.67 . Our findings indicate that the miR-181b rs322931 may be singly and jointly responsible for the etiology of SLE.

Some limitations should be discussed in the interpretation of our data. It is evident that nongenetic factors such as cigarette smoking, oxidative stress, ultraviolet light, infection, and hormonal action play important roles in the development of SLE [3-6]. In this study, we did not collect these factors and thus, the association of miR$181 \mathrm{~b}$ and IFNA1 polymorphisms with environment factors cannot be analyzed. Additionally, the samples in this study were not large enough and all of them were enrolled from Han Chinese. Further studies with larger sample sizes are required to assess whether our positive findings can be confirmed in other ethnicities. 
In summary, we found for the first time that the rs322931 C risk allele was related to the development of SLE and the rs322931 CT/TT genotypes altered miR-181b expression levels in SLE patients. Of note, miR-181b-IFNA1 interaction conferred the risk of SLE. Once confirmed in other ethnicities with larger sample sizes, it is likely to be important for future personalized treatment of SLE by genotyping the miR-181b rs322931 and IFNA1 rs1332190 and rs10811543.

\section{Data Availability}

The digital data used to support the findings of this study are available from the corresponding author upon request.

\section{Additional Points}

Key-points. 1. Individuals with the miR-181b rs322931 CT, CT/TT, and T allele had an increased trend of SLE risk. 2. Combined analysis showed a significant association of rs322931-rs1332190 and rs322931-rs10811543 with SLE risk. 3. Gene-gene interaction analysis showed that the three-locus model consisting of rs322931, rs1332190, and rs10811543 attributed an increased risk of SLE. 4. The rs322931 CT/TT carriers displayed lower levels of miR-181b.

\section{Ethical Approval}

All procedures performed in studies involving human participants were in accordance with the ethical standards of the Institutional Review Board of the Affiliated Hospital of Guilin Medical University (2012022) and with the 1964 Helsinki declaration and its later amendments or comparable ethical standards.

\section{Consent}

Informed consent was obtained from all individual participants included in the study.

\section{Conflicts of Interest}

The authors declare that there are no conflicts of interest regarding the publication of this paper.

\section{Authors' Contributions}

He YL designed and wrote the manuscript. Yang J helped to perform experiments. Zeng Z performed statistical analysis. Shi X prepared figures. All authors reviewed the manuscript.

\section{Acknowledgments}

This study was funded by the Natural Science Foundation of Guangxi, China (No. 2018GXNSFAA138120).

\section{References}

[1] O. P. Rekvig, "Systemic lupus erythematosus: definitions, contexts, Conflicts, Enigmas," Frontiers in Immunology, vol. 9, p. $387,2018$.
[2] B. Tebbe and C. E. Orfanos, "Epidemiology and socioeconomic impact of skin disease in lupus erythematosus," Lupus, vol. 6, no. 2, pp. 96-104, 1997.

[3] C. B. Speyer and K. H. Costenbader, "Cigarette smoking and the pathogenesis of systemic lupus erythematosus," Expert Review of Clinical Immunology, vol. 14, no. 6, pp. 481-487, 2018.

[4] C. G. Parks, A. de Souza Espindola Santos, M. Barbhaiya, and K. H. Costenbader, "Understanding the role of environmental factors in the development of systemic lupus erythematosus," Best Practice \& Research Clinical Rheumatology, vol. 31, no. 3, pp. 306-320, 2017.

[5] M. Barbhaiya and K. H. Costenbader, "Environmental exposures and the development of systemic lupus erythematosus," Current Opinion in Rheumatology, vol. 28, no. 5, pp. 497505, 2016.

[6] C. G. Parks and A. J. De Roos, "Pesticides, chemical and industrial exposures in relation to systemic lupus erythematosus," Lupus, vol. 23, no. 6, pp. 527-536, 2014.

[7] K. H. Costenbader, S. Gay, M. E. Alarcon-Riquelme, L. Iaccarino, and A. Doria, "Genes, epigenetic regulation and environmental factors: which is the most relevant in developing autoimmune diseases?," Autoimmunity Reviews, vol. 11, no. 8, pp. 604-609, 2012.

[8] Y. Lu, H. Huang, C. Liu et al., "Association of S100B polymorphisms and serum S100B with risk of systemic lupus erythematous in a Chinese population," Genetics and Molecular Biology, vol. 42, no. 2, pp. 321-328, 2019.

[9] R. Wang, Y. L. Lu, H. T. Huang et al., "Association of interleukin 13 gene polymorphisms and plasma IL 13 level with risk of systemic lupus erythematosus," Cytokine, vol. 104, pp. 92-97, 2018.

[10] H. Xue, L. Gao, Y. Wu et al., "The IL-16 gene polymorphisms and the risk of the systemic lupus erythematosus," Clinica Chimica Acta, vol. 403, no. 1-2, pp. 223-225, 2009.

[11] R. Wang, Y. L. Zeng, H. M. Qin et al., "Association of interleukin 22 gene polymorphisms and serum IL-22 level with risk of systemic lupus erythematosus in a Chinese population," Clinical and Experimental Immunology, vol. 193, no. 2, pp. 143$151,2018$.

[12] H. T. Huang, J. M. Chen, J. Guo, Y. Lan, and Y. S. Wei, "The association of interleukin-31 polymorphisms with interleukin-31 serum levels and risk of systemic lupus erythematosus," Rheumatology International, vol. 36, no. 6, pp. 799805, 2016.

[13] H. Kaga, A. Komatsuda, A. Omokawa et al., "Downregulated expression of miR-155, miR-17, and miR-181b, and upregulated expression of activation-induced cytidine deaminase and interferon- $\alpha$ in PBMCs from patients with SLE," Modern Rheumatology, vol. 25, no. 6, pp. 865-870, 2015.

[14] H. S. Li, Y. Ning, S. B. Li et al., "Expression and clinical significance of miR-181a and miR-203 in systemic lupus erythematosus patients," European Review for Medical and Pharmacological Sciences, vol. 21, no. 21, pp. 4790-4796, 2017.

[15] T. K. Motawi, D. A. Mohsen, S. A. El-Maraghy, and M. A. Kortam, "MicroRNA-21, microRNA-181a and microRNA-196a as potential biomarkers in adult Egyptian patients with systemic lupus erythematosus," Chemico-Biological Interactions, vol. 260, pp. 110-116, 2016.

[16] A. L. Carlsen, A. J. Schetter, C. T. Nielsen et al., "Circulating microRNA expression profiles associated with systemic lupus 
erythematosus," Arthritis and Rheumatism, vol. 65, no. 5, pp. 1324-1334, 2013.

[17] A. Kozomara, M. Birgaoanu, and S. Griffiths-Jones, "miRBase: from microRNA sequences to function," Nucleic Acids Research, vol. 47, no. D1, pp. D155-D162, 2019.

[18] D. P. Bartel, "MicroRNAs: genomics, biogenesis, mechanism, and function," Cell, vol. 116, no. 2, pp. 281-297, 2004.

[19] Y. A. Lashine, A. M. Seoudi, S. Salah, and A. I. Abdelaziz, "Expression signature of micro RNA-181-a reveals its crucial role in the pathogenesis of paediatric systemic lupus erythematosus," Clinical and Experimental Rheumatology, vol. 29, no. 2, pp. 351-357, 2011.

[20] E. R. Hutchison, E. M. Kawamoto, D. D. Taub et al., "Evidence for miR-181 involvement in neuroinflammatory responses of astrocytes," Glia, vol. 61, no. 7, pp. 1018-1028, 2013.

[21] Y. Wang, G. Mao, Y. Lv, Q. Huang, and G. Wang, "MicroRNA-181b stimulates inflammation via the nuclear factor- $\kappa \mathrm{B}$ signaling pathway in vitro," Experimental and Therapeutic Medicine, vol. 10, no. 4, pp. 1584-1590, 2015.

[22] S. Ghorbani, F. Talebi, W. F. Chan et al., "MicroRNA-181 variants regulate $\mathrm{T}$ cell phenotype in the context of autoimmune neuroinflammation," Frontiers in Immunology, vol. 8, p. 758, 2017.

[23] F. Yuan, R. Sun, L. Li et al., "A functional variant rs353292 in the flanking region of miR-143/145 contributes to the risk of colorectal cancer," Scientific Reports, vol. 6, no. 1, p. 30195, 2016.

[24] R. Sun, Y. Liang, F. Yuan et al., "Functional polymorphisms in the promoter region of miR-17-92 cluster are associated with a decreased risk of colorectal cancer," Oncotarget, vol. 8, no. 47, pp. 82531-82540, 2017.

[25] F. Yuan, X. Xiao, G. Che et al., "A functional variant in the flanking region of pri-let-7f contributes to colorectal cancer risk in a Chinese population," Journal of Cellular Physiology, vol. 234, no. 9, pp. 15717-15725, 2019.

[26] R. Zhang, B. Pan, Y. Li, and X. Li, "SNP rs4937333 in the miRNA-5003-Binding site of the ETS1 3'-UTR decreases ETS1 expression," Frontiers in Genetics, vol. 10, p. 581, 2019.

[27] C. Ciccacci, C. Perricone, C. Politi et al., "A polymorphism upstream MIR1279 gene is associated with pericarditis development in systemic lupus erythematosus and contributes to definition of a genetic risk profile for this complication," Lupus, vol. 26, no. 8, pp. 841-848, 2017.

[28] L. Fu, L. Jin, L. Yan et al., "Comprehensive review of genetic association studies and meta-analysis on miRNA polymorphisms and rheumatoid arthritis and systemic lupus erythematosus susceptibility," Human Immunology, vol. 77, no. 1, pp. 1-6, 2016.

[29] A. P. Wingo, L. M. Almli, J. S. Stevens et al., "Genome-wide association study of positive emotion identifies a genetic variant and a role for microRNAs," Molecular Psychiatry, vol. 22, no. 5, pp. 774-783, 2017.

[30] X. Han, Z. Zheng, C. Wang, and L. Wang, "Association between MEG3/miR-181b polymorphisms and risk of ischemic stroke," Lipids in Health and Disease, vol. 17, no. 1, p. 292, 2018.

[31] M. C. Hochberg, "Updating the American College of Rheumatology revised criteria for the classification of systemic lupus erythematosus," Arthritis and Rheumatism, vol. 40, no. 9, p. $1725,1997$.
[32] J. Zhao, H. Wu, M. Khosravi et al., “Association of genetic variants in complement factor $\mathrm{H}$ and factor $\mathrm{H}$-related genes with systemic lupus erythematosus susceptibility," PLoS Genetics, vol. 7, no. 5, article e1002079, 2011.

[33] K. J. Livak and T. D. Schmittgen, "Analysis of relative gene expression data using real-time quantitative PCR and the 2(-Delta Delta C (T)) method," Methods, vol. 25, no. 4, pp. 402-408, 2001.

[34] Y. Y. Shi and L. He, "SHEsis, a powerful software platform for analyses of linkage disequilibrium, haplotype construction, and genetic association at polymorphism loci," Cell Research, vol. 15, no. 2, pp. 97-98, 2005.

[35] L. W. Hahn, M. D. Ritchie, and J. H. Moore, "Multifactor dimensionality reduction software for detecting gene-gene and gene-environment interactions," Bioinformatics, vol. 19, no. 3, pp. 376-382, 2003.

[36] H. Li and G. Ding, "Elevated serum inflammatory cytokines in lupus nephritis patients, in association with promoted hsamiR-125a," Clinical Laboratory, vol. 62, no. 4, pp. 631-638, 2016.

[37] H. Zhang, X. Huang, L. Ye et al., "B cell-related circulating MicroRNAs with the potential value of biomarkers in the differential diagnosis, and distinguishment between the disease activity and lupus nephritis for systemic lupus erythematosus," Frontiers in Immunology, vol. 9, p. 1473, 2018.

[38] T. M. Lancaster, N. Ihssen, L. M. Brindley, and D. E. J. Linden, "Further support for association between GWAS variant for positive emotion and reward systems," Translational Psychiatry, vol. 7, no. 1, article e1018, 2017.

[39] P. G. Bronson, C. Chaivorapol, W. Ortmann, T. W. Behrens, and R. R. Graham, "The genetics of type I interferon in systemic lupus erythematosus," Current Opinion in Immunology, vol. 24, no. 5, pp. 530-537, 2012.

[40] S. Oon, N. J. Wilson, and I. Wicks, "Targeted therapeutics in SLE: emerging strategies to modulate the interferon pathway," Clinical \& Translational Immunology, vol. 5, no. 5, article e79, 2016.

[41] S. M. Abdel Galil, A. M. El-Shafey, R. S. Abdul-Maksoud, and M. El-Boshy, "Interferon alpha gene expression and serum level association with low vitamin D levels in Egyptian female patients with systemic lupus erythematosus," Lupus, vol. 27, no. 2, pp. 199-209, 2018.

[42] P. Blanco, A. K. Palucka, M. Gill, V. Pascual, and J. Banchereau, "Induction of dendritic cell differentiation by IFN-alpha in systemic lupus erythematosus," Science, vol. 294, no. 5546, pp. 1540-1543, 2001.

[43] K. A. Kirou, C. P. Mavragani, and M. K. Crow, "Activation of type I interferon in systemic lupus erythematosus," Expert Review of Clinical Immunology, vol. 3, no. 4, pp. 579-588, 2007.

[44] A. Le Bon, C. Thompson, E. Kamphuis et al., "Cutting edge: enhancement of antibody responses through direct stimulation of B and T cells by type I IFN," Journal of Immunology, vol. 176, no. 4, pp. 2074-2078, 2006.

[45] K. Gkirtzimanaki, E. Kabrani, D. Nikoleri et al., "IFN $\alpha$ Impairs Autophagic Degradation of mtDNA Promoting Autoreactivity of SLE Monocytes in a STING-Dependent Fashion," Cell Reports, vol. 25, no. 4, pp. 921-933.e5, 2018, e925.

[46] S. G. Thacker, C. C. Berthier, D. Mattinzoli, M. P. Rastaldi, M. Kretzler, and M. J. Kaplan, "The detrimental effects of IFN- $\alpha$ on vasculogenesis in lupus are mediated by repression of IL-1 pathways: potential role in atherogenesis and renal vascular rarefaction," Journal of Immunology, vol. 185, no. 7, pp. 4457-4469, 2010. 